\title{
Miscellany *
}

\section{Reviews of United Kingdom Statistical Sources}

Since 1969 work has been underway in the United Kingdom to compile a comprehensive series of Reviews of UK Statistical Sources under the joint sponsorship of the Economic and Social research Council (formerly the Social Science Research Council) and the Royal Statistical Society. Twenty volumes (covering thirty-six review topics) have now been completed. This note provides an account of the scope and nature of the series and of the topics covered in the first twenty volumes.

The series was conceived as a successor to one produced under the auspices of the Royal Statistical Society and edited by Maurice Kendall (later Sir Maurice Kendall) in the early post-war years and published under the title The Sources and Nature of the Statistics of the United Kingdom (kendall, 1952 and 1957). These two volumes provided a series of critical reviews of selected areas of economic and social statistics written by many of the foremost national experts of the time. By the late 1960s, however, not only had this series become badly out of data but the field had been completely transformed by a very considerable growth not only in regular statistical reporting but also in the use of ad hoc statistical enquiries in many fields by both official and unofficial agencies. These developments meant that a new series of reviews was required and that they would have to be conceived on a much bigger scale. Since its inception in 1969 the new series has been guided by a steering committee drawn from the ESRC and the Royal Statistical Society and has been under the editorship of Professor W.F. Maunder throughout.

The original intention was to try to cover every field of economic and social statistics and to publish pairs of reviews on cognate topics in single volumes. This intention has been adhered to in most of the volumes published to data but five reviews have appeared as single volumes and, in order to speed the publication process, it is intended that future reviews will also appear as single volumes.

The purpose of the series is twofold. The primary aim is to act as an authoritative work of reference to the sources of statistical material of all kinds, both official and unofficial. Secondly, the series seeks to provide a critical appraisal of the nature and limitations of the available data so that the user is able to interpret the data safely and avoid pitfalls in their use.

The intention is that the source for each topic be reviewed in detail, and the brief supplied to authors has called for comprehensive coverage at the level of

* This section contains information about major statistical activities, which are either ongoing or were recently completed in countries. 
'national interest'. This term does not denote any necessary restriction to statistics collected on a national basis (still less, of course, to national aggregates) but it means that sources of a purely local character, without wider interest in either content or methodology, are excluded. Data are regarded as available not only if published in the normal printed format but also if they are likely to be released to a bona fide enquirer in any other form. On the other hand, no reference is made to material which even if it is known to exist, is not accessible to the general run of potential users.

To facilitate the use of the series as a work of reference certain standard features are incorporated. First, the text of each review is designed, insofar as varying subject matter permits, to follow a standard form of arrangement so that users may expect a similar pattern to be followed throughout the series. The starting point is a brief summary of the activity concerned and its organisation, in order to give a clear background understanding of how data are collected, what is being measured, the stage at which measurements are made, what the reporting units are, the channels through which returns are routed and where they are processed. As a further part of this introductory material, there is a discussion of the specific problems of definition and measurement to which the topic gives rise. The core sections on available sources which follow are arranged, at the author's discretion, by origin, by subject subdivision, or by type of data etc. A final chapter in each review is devoted to a discussion of general shortcomings and desirable improvements. In case contrary expectations should be aroused, it should be said that authors have not been asked to produce a comprehensive plan for the reform of statistical reporting in the whole of their field. However, a review of existing sources is a natural opportunity to make some suggestions for future policy on the collection and publication of statistics within the field concerned and authors have been encouraged to take full advantage of it.

Secondly, detailed factual information about statistical series and other data is given in a Quick Reference List (QRL). This lists all the available data very specifically within subject groupings giving information about the categories of data, sub-classification, periods of time covered, frequency etc. together with cross-references to the section of the text in which the data are discussed.

Thirdly, a subject index to each review acts as a more or less conventional line of enquiry on textual references. But an important feature is that it is a computerised system. For an individual review this introduces the possibility of easy permutation of entries and this is fully utilised in order to facilitate search by giving as many variants as possible. Taken over all reviews the system makes possible selective searches by key word over any combination of reviews and a print-out of the entries found may then be prepared. Anyone wishing to use this facility is invited to apply to the editorial office at Exeter University.

Fourthly, each review contains two listings of publications. A $Q R L$ Key gives full details of the publications shown as data sources. The other listing is a general Bibliography. This gives references to works discussing wider aspects such as methodology and uses of the data.

Finally, specimen copies of the more important returns or forms and question- 
Table 1

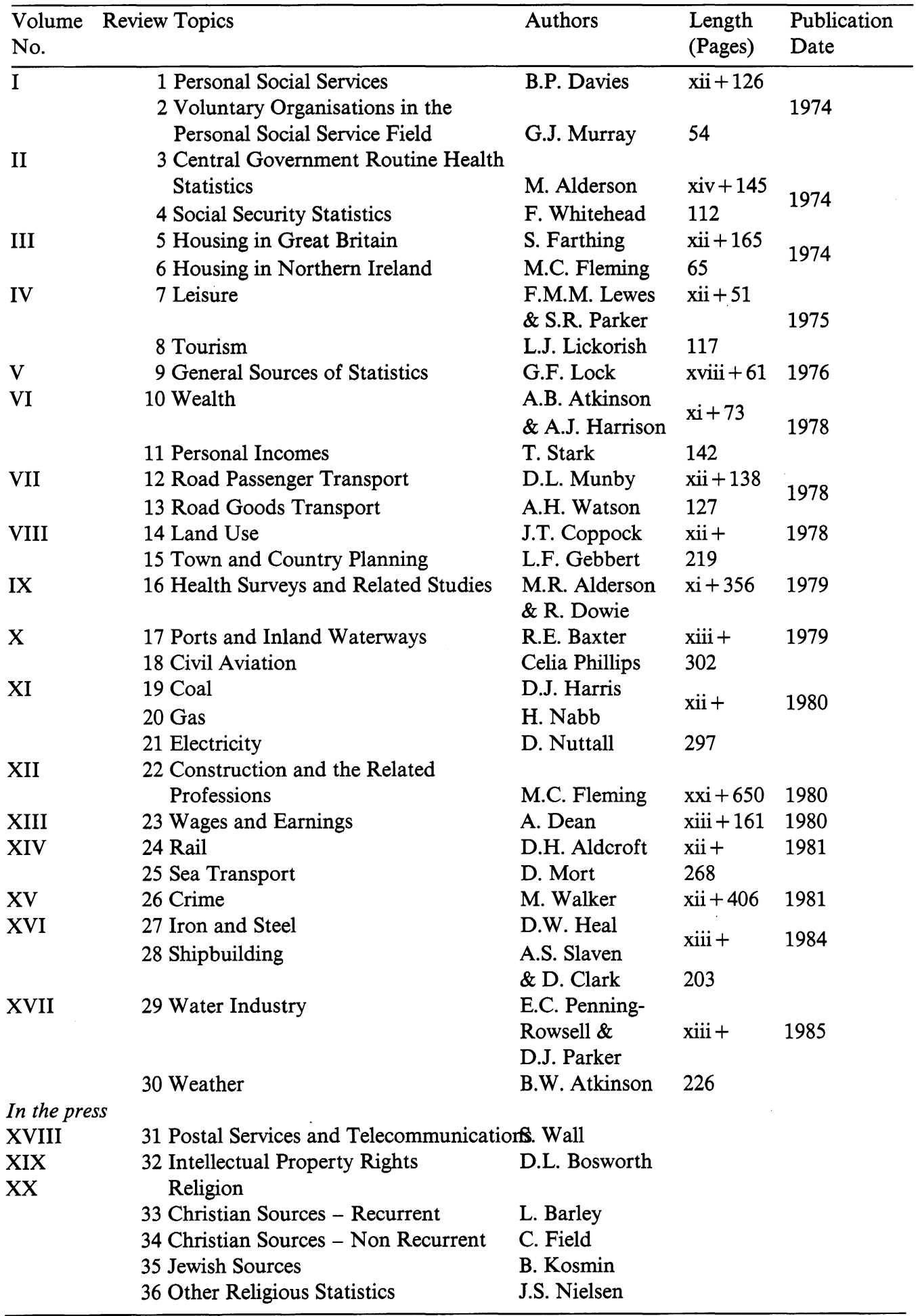


naires used in data collection are reproduced so that it may be seen what tabulations it is possible to make as well as helping to clarify the basis of those actually available.

The topics covered in the first 20 volumes together with publication details are given in the Table 1 below.

Volumes expected to go into production shortly include Financial Institutions, Local Government, Newspaper and Periodicals, Printing and Publishing and Agriculture.

Professor W.F. Maunder University of Exeter

Dr. M.C. Fleming Loughborough University of Technology 